\title{
A MICROMACHINED PENDULOUS OSCILLATING GYROSCOPIC ACCELEROMETER
}

\author{
Todd J. Kaiser \\ Milli Sensor Systems \& Actuators, Inc. \\ West Newton, MA 02165
}

\section{ABSTRACT}

A silicon Pendulous Oscillating Gyroscopic Accelerometer (POGA) was fabricated using deep reactive ion etching (DRIE) and silicon wafer bonding technologies. The accelerometer is composed of three individual layers that are assembled into the final instrument. The top layer uses wafer bonding of an oxidized wafer to a handling wafer to create a silicon-on-oxide wafer pair, in which the oxide layer provides electrical isolation between the mechanical members and the handling layer. The middle layer is a two-gimbal torsionallysupported silicon structure, and is in turn supported by an underlying drive/sense layer. The instrument proved to have better than milli-g resolution and dynamic ranges in excess of $1-g$ (open loop) and approximately 12 milli-g (closed loop).

\section{INTRODUCTION}

The Pendulous Oscillating Gyroscopic Accelerometer (POGA) is the oscillatory analog of the Pendulous Integrating Gyro Accelerometer (PIGA), the most accurate strategic-grade accelerometer to date [1]. Instead of rotating members as in the PIGA, the members of the three orthogonal axis system in the POGA oscillate [2]. The interaction of the oscillations of the inner and outer members provide a DC torque to the middle member to rebalance a pendulous seismic mass. Because the members oscillate rather than rotate, significant design and manufacturing simplifications are possible. The oscillatory nature of the POGA makes it amenable to layered fabrication, which is achievable using micromachining technologies. The operation of the POGA is easiest understood if the operation of the PIGA is first reviewed.

The PIGA operation is based on gyroscopic theory of rotating bodies [3-5]. A spinning mass called the momentum wheel or rotor generates angular momentum about the spin axis. The rotor is supported by a housing called the torque-summing member (TSM) that allows rotation of the wheel about a second axis that is perpendicular to the spin axis. This combination of components is a single-degree-of-freedom gyroscope. A rotation of the gy ro about a third axis that is perpendicular to both the TSM and rotor axes, generates a gyroscopic torque on the TSM which acts to rotate the TSM. The third axis is usually referred to as the input axis and the TSM axis is usually referred to as the output axis.

A PIGA is formed from the above gyroscope by making the TSM pendulous and by mounting the gyro onto a member that allows the rotation of the gyro about its input axis. This member is referred to as the Servo Driven Member (SDM). The TSM is made pendulous by adding a mass, $m$, to the TSM usually along the spin axis at some moment arm, $l$, from the output axis. The result is an arrangement that sums a pendulous and gyroscopic torque on the same member, the TSM. Since acceleration varies the pendulous torque, the gyroscopic torque can be servoed by rotating the SDM to balance the pendulum

\author{
Mark G. Allen \\ School of Electrical and Computer Engineering \\ Georgia Institute of Technology \\ Atlanta, GA 30332-0250
}

and hence hold the TSM at null. At this null, the rotation of the SDM can be related to the input acceleration. The equilibrium torque equation is given by:

$$
m l a=I, \dot{\psi} \dot{\phi},
$$

where $I_{1}$ is the moment of inertia of the rotor about the spin axis, $\dot{\psi}$ is the angular velocity of the rotor, and $\dot{\phi}$ is the angular velocity of the SDM.

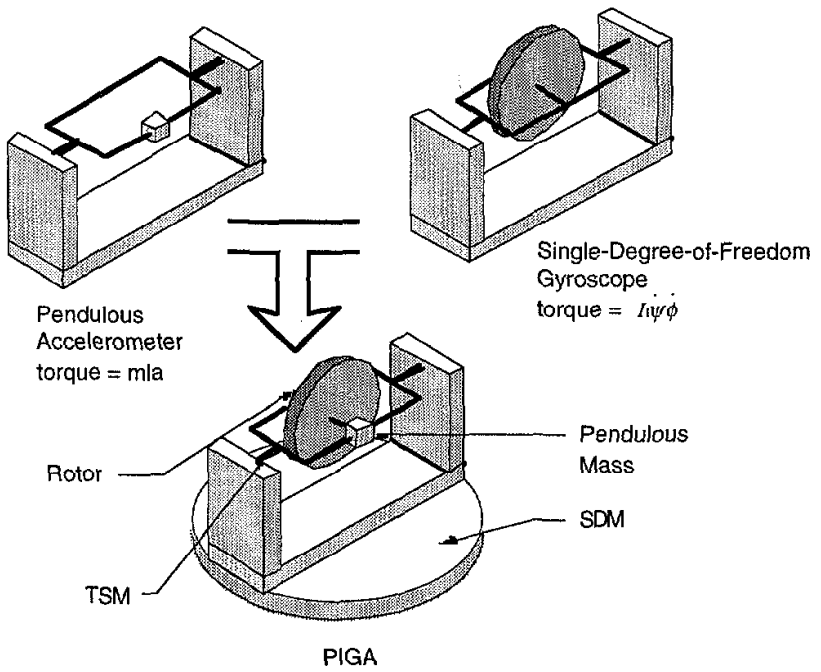

Figure 1. The PIGA is the combination of a pendulous accelerometer and a single-degree-of-freedom gyroscope.

This replacement of the rotary members with oscillatory members is the fundamental design change between the PIGA and the POGA. It makes the device suitable for microfabrication while maintaining the fundamental physics that has produced the highest performing accelerometers.

The POGA is also a three orthogonal axis system. Instead of rotating members as in the PIGA, the members are supported by flexures and designed to oscillate. The inner member designated the rotor-driven member (RDM) generates the angular momentum just as in the PIGA, but instead of a fixed angular momentum, the angular momentum oscillates. The oscillating angular momentum requires an oscillating SDM to generate the gyroscopic torque that nulls the TSM. If the RDM and SDM are forced to oscillate at the same frequency $\omega$, then the equilibrium torque equation can be given by:

$$
m l a=I_{1} \dot{\psi} \dot{\phi}=I_{1} \Psi \Phi \omega^{2} \sin (\omega t) \sin (\omega t+\beta),
$$

where $\Psi$ and $\Phi$ are the amplitude of oscillation of the RDM and SDM, and $\beta$ is phase difference between their oscillations. 
Trigonometric and algebraic manipulations produce the resulting expression for the acceleration:

$$
a=\frac{I_{1} \Psi \Phi}{2 m l} \omega^{2} \cos \beta+f(2 \omega)
$$

The acceleration is a function of the cosine of the phase difference between the two oscillations plus a second harmonic term that time averages to zero. The phase difference becomes the control signal used to maintain the TSM at null in a closedloop POGA.

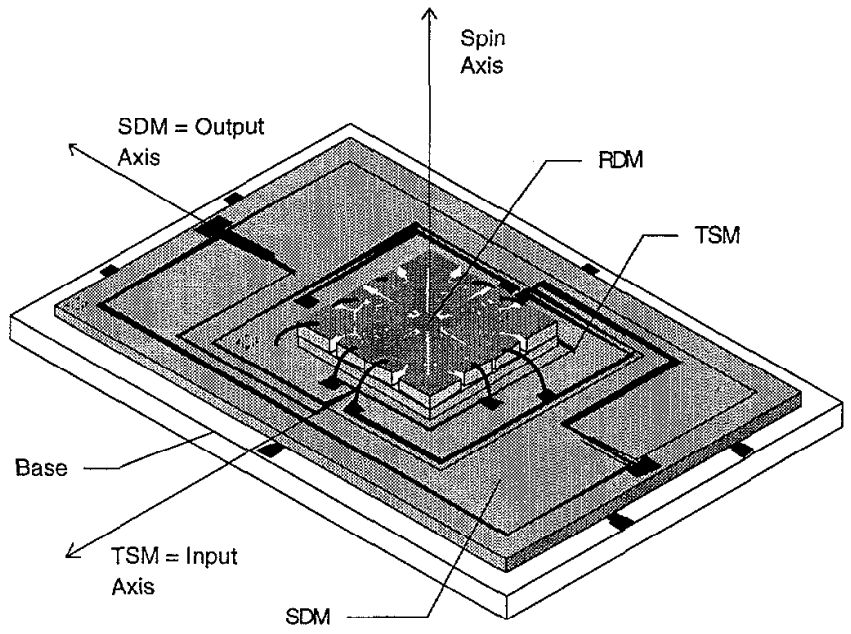

Figure 2. Schematic drawing of the micromachined POGA, showing the principal components and rotational axes.

\section{DEVICE DESCRIPTION}

The RDM of the micromachined POGA is a $4.5 \mathrm{~mm}$ diameter silicon member formed by etching entirely through a two inch wafer. It has two sets of four rotational electrostatic comb drives for in-plane actuation. The RDM is supported by four 50 micron wide silicon flexures that are anchored to an under layer of silicon. The spacing between the comb fingers is 50 microns. The comb drives and anchors are electrically isolated from each other by thermally grown silicon dioxide.
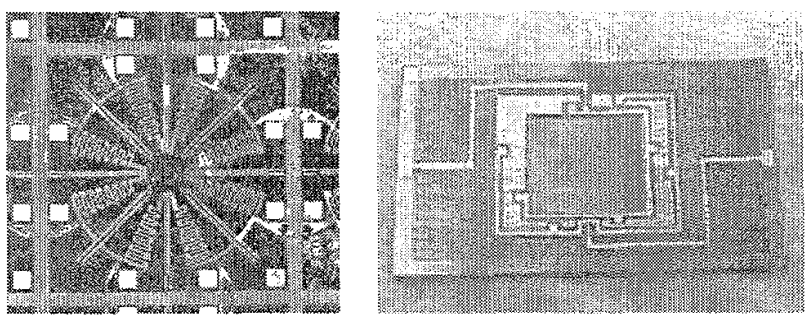

Figure 3. The left image shows a single RDM in an array of devices. Each RDM has a diameter of 4.5 millimeters and is formed on a $6 \times 6 \mathrm{~mm}$ silicon die connected by tabs. The right image is an SDM/TSM assembly with a recess for mounting the RDM an the TSM. Aluminum conduits isolated by an oxide layer run on the surface. The SDM frame measures $13 \times 20 \mathrm{~mm}$.

The TSM and the SDM are silicon members etched from the same 2 inch silicon wafer as a single unit. The TSM is inset within the SDM. The TSM flexures are 50 microns wide and 1 $\mathrm{mm}$ long connecting the TSM to the SDM. The SDM flexure dimensions are varied to match the SDM resonant frequency to the RDM resonant frequency. These flexures attach the SDM to a frame that is mounted to a supporting base. The SDM has 100 micron holes perforating the silicon structure to reduce the squeeze film damping between the SDM and the supporting glass base. The TSM requires damping to reduce the vibration sensitivity of the TSM, so no perforations are necessary. The back side of the SDM and TSM are etched to create the necessary gap to allow motion of these members. This gap was varied between 10 and 50 microns. Smaller gaps required less drive voltages but allow smaller mechanical motion. The front side has electrical conduits patterned on the surface separated from the substrate by a layer of silicon dioxide.
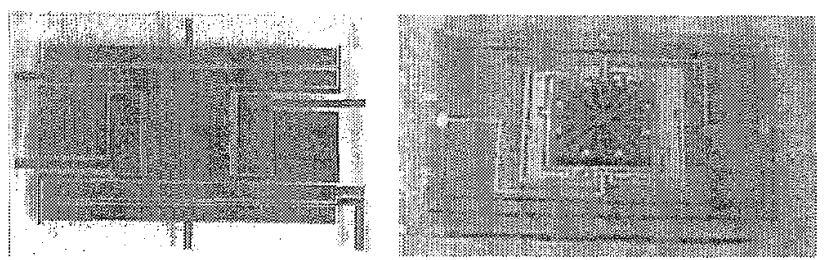

Figure 4. The left image shows the base electrode configuration The right image is an assembled POGA: RDM mounted on TSM, SDM mounted on base, $15 \times 22 \mathrm{~mm}$.

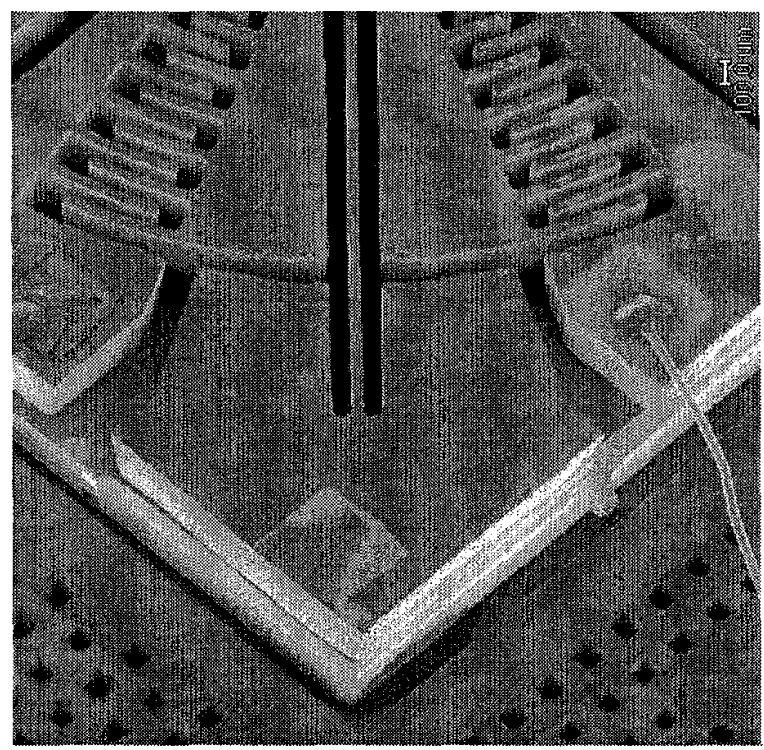

Figure 5. SEM of a quadrant of RDM. The RDM flexure runs vertically in the image between the radial comb drives. A wire bond from the comb drive is visible on the right side of the image. The edges of the two bonded layers and the perforations in the SDMTTSM layer are visible.

Figure 3 shows an RDM still in a device array on the wafer. Small tabs are left during the etching process to interconncet the components. These arc casily clcaved to dice out the individual components. The second image in Figure 3 is an SDM/TSM assembly. The SDM axis of rotation is horizontal in the image with electrical connections running to the perimeter. The TSM axis of rotation is vertical in the image with the electrical conduit running across the surface of the flexures.

The SDM/TSM assembly is mounted on a base that provides electrodes for actuation and sensing of the mechanical members. The drive electrodes for the SDM are positioned near the edge of the member to increase the torque generated. The 
SDM sensing electrodes are positioned just inside the drive electrodes. The TSM sensing electrodes are placed under the TSM at the edge of the member to maximize sensitivity to rotations. Both sensor systems use differential capacitor readout electronics to monitor the position of the SDM and TSM. A ground plane is serpentined between the electrodes to reduce cross-talk between the capacitors. Figure 4 shows an image of the base with the electrode layout visible. The second image of Figure 4 is an assembled POGA. The RDM mounts on the TSM. The RDM itself creates the pendulosity of the TSM.

A scanning electron microscope image of an assembled POGA is shown in Figure 5. Due to the relatively large size of the device, only a portion of the instrument is visible. The image is an isometric view of the RDM mounted on the TSM.

\section{FABRICATION PROCESS}

The micromachined POGA is fabricated in three separate assemblies, the RDM, the SDM/TSM assembly and the base. The RDM and SDM/TSM assembly were etched out of silicon wafers in a Plasma Therm inductively coupled plasma reactive ion etcher (ICP-RIE). Characterization and discussion of the procedure can be found elsewhere [6]. The base is formed in glass with patterned metal electrodes on the surface.

The RDM fabrication sequence is shown in Figure 6. A two inch p-type silicon wafer is oxidized to produce a one micron layer of oxide surrounding the wafer. The oxidized wafer is bonded to another non-oxidized wafer using standard techniques [7]. The bonded wafers are now electrically isolated by the oxide layer between them. The wafers are then oxidized. The top wafer is then patterned and etched down to the buried oxide layer defining the RDM, its flexures, and its radial comb drives. The wafer combination is then etched from the backside to release the RDM mechanical structure, yet retain the anchors for the flexures and comb drive. The oxide is then removed from the exterior surfaces and aluminum is patterned on the comb drive anchors by using a shadow mask.

The shadow masks were also produced using the ICP. Holes were etched through silicon wafers to produce the masks. When the mask is aligned to the wafer, aluminum was allowed to deposit on the component only in the required areas. The shadow masks were produced for both device level metalization and wafer level metalization.

The fabrication sequence of the SDM/TSM assembly is shown in Figure 7. A p-type two inch silicon wafer is oxidized to produce a one micron layer of oxide. Windows are etched in the oxide to provide contact regions to the silicon. Aluminum is deposited on the wafer, sintered then patterned. The backside of the wafer is etched to produce the standoff height between the base metal layers and the silicon mechanical layers. The wafer is then etched entirely through its thickness from the front side to define the SDM and TSM.

The bases are formed in glass. Aluminum is deposited on the glass with a titanium adhesion layer. The metals are then patterned to produce the SDM drive electrodes, and the sensing electrodes for the SDM and TSM.

The three components are then assembled to form the instrument. The assembly procedure is shown in Figure 8 . The SDM/TSM assembly is temporarily mounted on a mesa support structure. The mesa is used to handle the component and support the movable members during assembly. The RDM is positioned on the TSM and epoxied in place. A recess in the TSM aids in the alignment of the RDM to the TSM. Electrical connections are made from the RDM by wirebonding. The SDM/TSM assembly is released from the supporting mesa and epoxied to the glass base. The base is then wirebonded to the package.

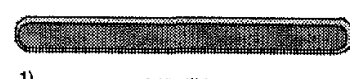

1)

Oxidizo Water
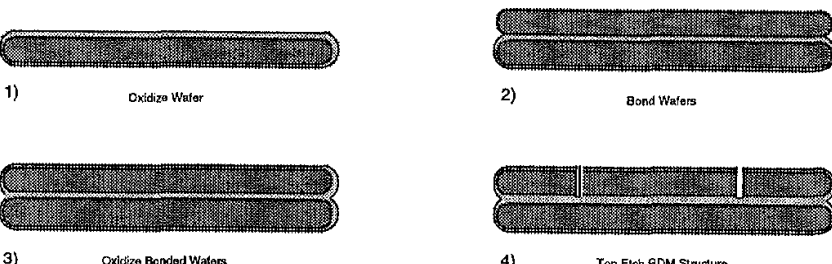

3) Oxlcizo Bendod Wators

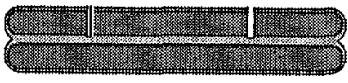

Top Etch ADM Structurs

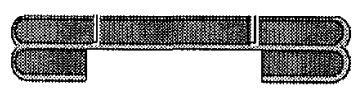

5) Bottom Ekch Rolleaso

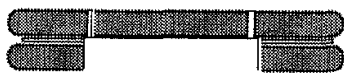

6) Elch Oxido

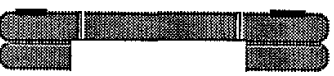

Shadow Maek Metalt

Figure 6. RDM fabrication sequence.
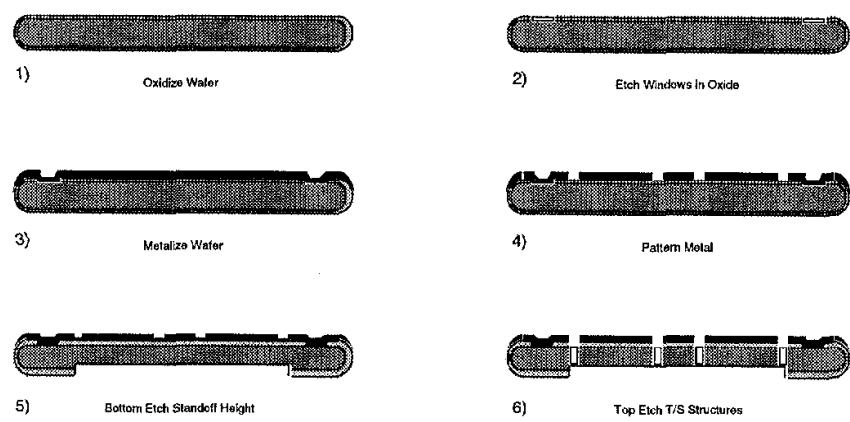

6) Top Ekch T/S Structure

Figure 7. SDM/TSM assembly fabrication sequence.
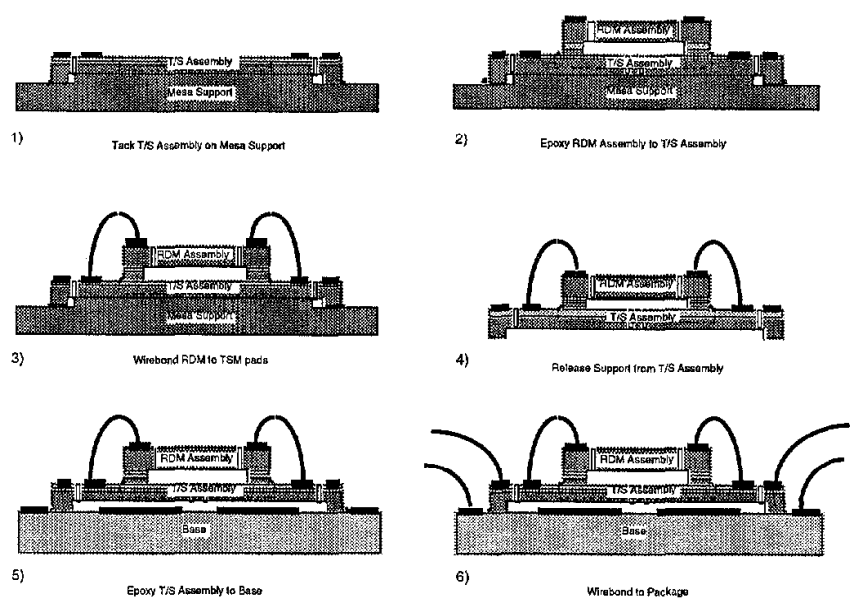

Figure 8. Micromachined POGA assembly procedure.

\section{RESULTS}

Open loop, the POGA operates just like any other pendulous accelerometer. Under acceleration, the pendulous mass torques the member against the supporting flexures. This motion is detected with the differential plate capacitors for the TSM. Figure 9 shows an open loop tumble test of the bulk micromachined POGA and an ADXL150, an Analog Devices 
$50 \mathrm{~g}$ micromachined accelerometer. The instruments are mounted on a dividing head and rotated at $10^{\circ}$ steps through $360^{\circ}$, measuring the component of the earth's gravity along the input axis of the accelerometers.

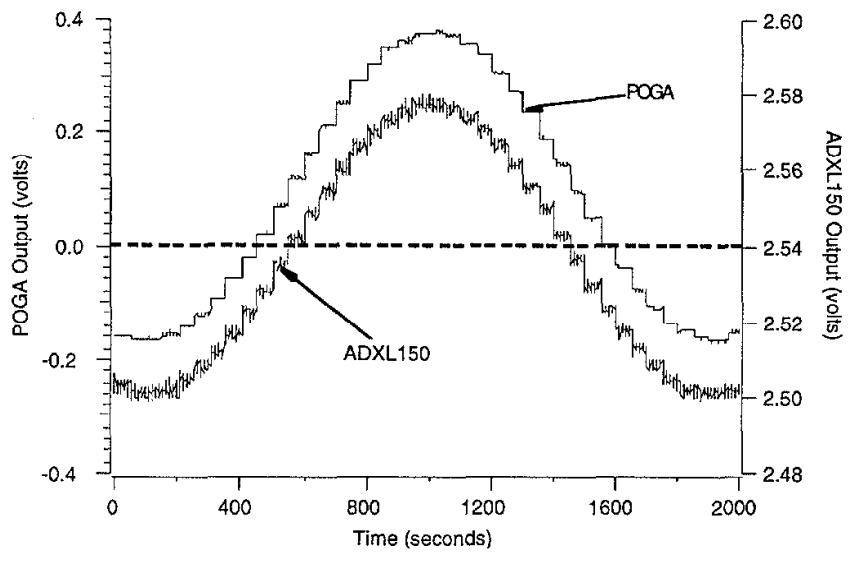

Figure 9. Open loop tumble test of $A D X L 150$ and micromachined POGA. Rotated $10^{\circ}$ steps at 50 second intervals measuring a component of earth's gravity.

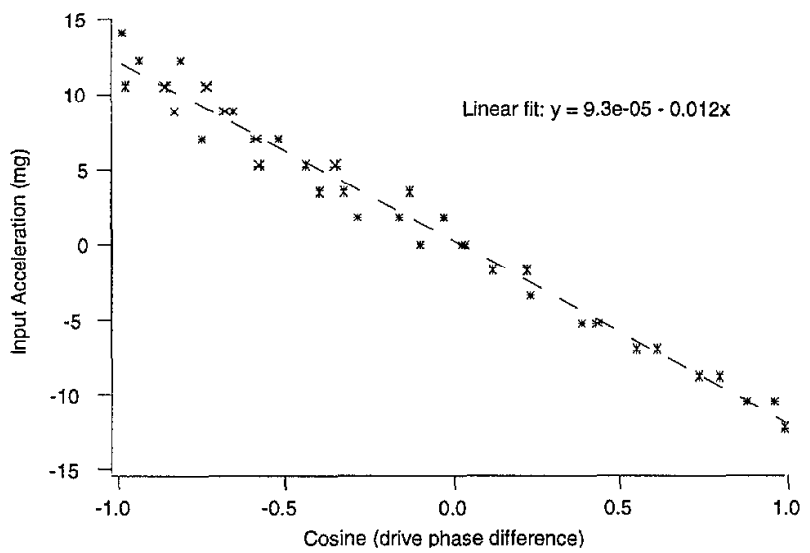

Figure 10. Closed loop scale factor test. Input acceleration nulled by adjusting phase between input drive voltages.

Closed loop, the POGA operation differs from other accelerometers. The innermost member, the RDM, generates an oscillating angular momentum. The drive frequency is set at the resonant frequency of the RDM. A bias voltage is applied to the sinusoidal signal to offset the drive so that it is always applying a positive potential to remove the frequency doubling effect of capacitive electrostatic actuators. The oscillating angular momentum is the functional equivalent to the constant angular momentum of a spinning wheel gyro. In the PIGA, the pendulous mass is torqued back to the null position by spinning the gyro to generate the gyroscopic torque. Similarly, the SDM of the POGA oscillates to create a torque that nulls the TSM. The drive voltage of the SDM is phase locked to the RDM drive. A bias voltage is also applied to remove frequency doubling effects. By controlling the relative phase and magnitude of the RDM and SDM oscillations, the middle pendulous torque summing member can be held at null.

Figure 10 shows the results of the closed loop scale factor test. The POGA was positioned such that the input axis of the accelerometer was vertical on a dividing head. The dividing head was then rotated to produce an input acceleration. The input acceleration was sensed by the TSM causing a shift in the output voltage. The voltage was then returned to the null position by adjusting the phase difference between the phase locked function generators used to drive the RDM and SDM. The input acceleration was calculated by taking the sine of the angle on the dividing head. Equation (3) shows the input acceleration should be a linear function of the cosine of the phase, experimentally confirmed in Figure 10.

\section{SUMMARY AND CONCLUSIONS}

The POGA uses the same fundamental physics that has been demonstrated to produce the highest performing accelerometer, the PIGA. The scale factor is determined only by mechanical quantities, the pendulosity and angular momentum, rather than by a precision voltage supply as in other servoed accelerometers.

The fabrication methods demonstrate the use of the ICP as a machining tool in addition to a micromachining tool. Application specific layers were produced then assembled into an instrument. The stacked layer design in conjunction with bulk micromachining is ideally suited for modern inertial sensors where reduced cost and high performance are required.

\section{ACKNOWLEDGMENTS}

Microfabrication was carried out in the Georgia Tech Microelectronics Research Center, with the assistance of the staff. The authors would also like to thank the staff of Milli Sensor Systems and Actuators for their numerous contributions as well as discussions of capacitive sensing.

The authors would like to acknowledge the Special Projects Office of the Navy, SP-23, who funded the early effort to demonstrate the POGA proof-of-principle model with conventional construction and subsequently motivated the demonstration of the silicon micromachined POGA.

\section{REFERENCES}

1. M. S. Sapuppo., "Pendulous Oscillating Gyro Accclerometcr; POGA," Joint Services Data Exchange for Guidance, Navigation \& Control, 24th, Anaheim, CA, Nov., 1998.

2. M. S. Sapuppo, "Pendulous Oscillating Gyro Accelerometer," U. S. Patent \#5,457,993, October 17, 1997.

3. A. Lawrence, Modern Inertial Technology, SpringerVerlag, New York, 1998.

4. G. R. Pitman, (Ed.), Inertial Guidance, Wiley, New York, 1962.

5. M. Fernandez and G. R. Macomber, Inertial Guidance Engineering, Prentice-Hall, Englewook Cliffs, NJ, 1962.

6. A. A. Ayon, R. Braff, C. C. Lin, H. H. Sawin, and M. A. Schmidt, "Characterization of a Time Multiplexed Inductively Coupled Plasma Etcher," J. Electrochem. Soc., Vol. 146, No. 1, pp. 339-349, 1999.

7. M. A. Schmidt, "Silicon Wafer Bonding for Micromechanical Devices," Technical Digest of the 1994 Solid State Sensor and Actuator Workshop, Hilton Head, SC, pp127131, 1994. 